\title{
Comparison of the Full Outline of UnResponsiveness and Glasgow Liege Scale/Glasgow Coma Scale in an Intensive Care Unit Population
}

\author{
Marie-Aurélie Bruno • Didier Ledoux • Bernard Lambermont • \\ François Damas • Caroline Schnakers • Audrey Vanhaudenhuyse • \\ Olivia Gosseries $\cdot$ Steven Laureys
}

Published online: 28 April 2011

(C) Springer Science+Business Media, LLC 2011

\begin{abstract}
Background The Full Outline of UnResponsiveness (FOUR) has been proposed as an alternative for the Glasgow Coma Scale (GCS)/Glasgow Liège Scale (GLS) in the evaluation of consciousness in severely brain-damaged patients. We compared the FOUR and GLS/GCS in intensive care unit patients who were admitted in a comatose state.

Methods FOUR and GLS evaluations were performed in randomized order in 176 acutely ( $<1$ month) braindamaged patients. GLS scores were transformed in GCS scores by removing the GLS brainstem component. Interrater agreement was assessed in $20 \%$ of the studied population $(N=35)$. A logistic regression analysis adjusted for age, and etiology was performed to assess the link between the studied scores and the outcome 3 months after injury $(N=136)$.

Results GLS/GCS verbal component was scored 1 in 146 patients, among these 131 were intubated. We found that
\end{abstract}

Marie-Aurélie Bruno and Didier Ledoux contributed equally to this study.

M.-A. Bruno - D. Ledoux - C. Schnakers - A. Vanhaudenhuyse · O. Gosseries $\cdot$ S. Laureys ( $\square)$

Coma Science Group, Cyclotron Research Centre and Neurology Department, University and University Hospital of Liège,

Sart-Tilman (B30), 4000 Liège, Belgium

e-mail: steven.laureys@ulg.ac.be

B. Lambermont

Intensive Care Department, Liège University Hospital and University of Liège, 4000 Liège, Belgium

F. Damas

Intensive Care Department, Centre Hospitalier Régional de la Citadelle, University of Liège, 4000 Liège, Belgium the inter-rater reliability was good for the FOUR score, the GLS/GCS. FOUR, GLS/GCS total scores predicted functional outcome with and without adjustment for age and etiology. 71 patients were considered as being in a vegetative/unresponsive state based on the GLS/GCS. The FOUR score identified 8 of these 71 patients as being minimally conscious given that these patients showed visual pursuit.

Conclusions The FOUR score is a valid tool with good inter-rater reliability that is comparable to the GLS/GCS in predicting outcome. It offers the advantage to be performable in intubated patients and to identify non-verbal signs of consciousness by assessing visual pursuit, and hence minimal signs of consciousness ( $11 \%$ in this study), not assessed by GLS/GCS scales.

Keywords Coma - Full Outline of UnResponsiveness . Glasgow Coma Scale · Glasgow Liège Scale . Vegetative state $\cdot$ Minimally conscious state . Neurological assessment - Intensive care

\section{Introduction}

Following severe brain damage and coma, some patients may awaken (i.e., open the eyes) but remain unresponsive (i.e., only showing reflex movements). This clinical syndrome is called vegetative/unresponsive state $[1,2]$. Patients who do recover, classically evolve to a minimally conscious state, defined by the presence of non-reflex voluntary movements such as orientation to pain, eye tracking, or reproducible albeit inconsistent command following. By definition, minimally conscious state patients cannot communicate their thoughts or wishes [3]. The clinical assessment of consciousness relies on 
disentangling automatic responses from non-reflexoriented movements or command following. This can be very challenging in coma and related disorders. Misdiagnosis can have clinical and therapeutic consequences, especially with regard to treatment of pain [4, 5]. Contrary to patients in vegetative/unresponsive state, those in minimally conscious state retain some capacity for cognitive, emotional, and pain processing [6-8]. Neurological assessment of those comatose patients and their outcome prediction are complex due to the difficulty of capturing distinct details of the clinical examination. The complexity of such assessment can also be explained by the difficulty of finding usable terminology permitting to describe the neurological status of a single patient. In recognition of these problems, scales have been constructed in an attempt to bring uniformity to the neurological examination and to standardize communication about the level of consciousness. The most commonly used scale is the Glasgow Coma Scale (GCS) [9, 10]. The GCS initially intended to assess the level of consciousness after head injury in neurosurgical intensive care unit is widely used in neurological patients beyond the original intentions in the context of outcome prediction (e.g., [11].), neurosurgical prognostic indicator (e.g., [12].), cerebral dysfunction measurement (e.g., [13].), and consciousness evaluation [14]. Over the years, considerable limitations have been identified for this scale: the inconsistent inter-observer reliability [15], the impossibility to test the verbal component in intubated patients $[16,17]$, the exclusion of the brainstem reflexes [18], the incapacity to detect subtle changes in neurological examination, and the lack of correlation between outcome and GCS scores [19]. Attempts have been made to modify the GCS [20-22]. In 1982, Born et al. [18] suggested that adding measures of brainstem reflexes to the GCS could improve prognostic information, but this scale-the Glasgow Liège Scale (GLS)—never had a widespread international use. Wijdicks et al. [23] recently presented a new coma scale named the Full Outline of UnResponsiveness (FOUR) as an alternative to the GCS/GLS in the evaluation of consciousness in severely brain-damaged patients. The FOUR score, contrary to the GLS/GCS, avoids assessing verbal function. Indeed, in the acute care setting, most patients are intubated or tracheotomized which makes accurate assessment of verbal responses difficult. It consists of four components: eyes and motor responses, brainstem reflexes, and respiration.

We here aimed to compare the FOUR score with the GLS/GCS, assessing inter-observer variability of the FOUR and comparing outcome prediction and diagnostic accuracy of the different coma scales (i.e., identification of vegetative/unresponsive [2] vs. minimally conscious states [3]).

\section{Method}

We prospectively assessed the FOUR and the GLS scores, in a randomized order, in adults consecutively admitted to five subunits of the medical and general intensive care departments of the Liège University Hospital and in one unit of the Citadelle Regional Hospital in Liège, Belgium. GLS scores were transformed to the more widely used GCS scores (GLS equals to GCS scores except for the addition of brainstem reflex assessment) [18]. Hence, GCS and GLS were not independent measures. Inclusion criteria were GCS $<8$ on admission and the absence of sedation or neuromuscular function blockers. Patients were assessed once within 1 month after the acute traumatic or nontraumatic brain injury. The assessments were performed by 1 ICU nurse (seen $N=36$ patients), 4 neuropsychologists (seen $N=115$ patients), 2 senior ICU specialists (seen $N=32$ patients), and 1 senior registrar (seen $N=28$ patients) who either had previous knowledge or had provided some type of care to these patients. Raters had established skill in scoring GLS/GLS, they were provided with a one-page written and visual instruction handout describing the FOUR score. These instructions were a French translation of the original instruction from the Mayo Clinic [23]. Raters were asked to grade a few patients using both GLS/GSC and FOUR scales prior the study. In intubated patients, the rating for the verbal domain of the GLS/GCS was defined to be 1 .

First, we assessed the association between GLS/GCS and FOUR scales using Spearman correlation coefficient with Bonferroni correction for multiple comparisons. Second, to investigate the reliability between examiners, weighted Cohen's kappa $\left(\kappa_{\mathrm{w}}\right)$ values were used to determine the reproducibility of FOUR, GCS, and GLS total and sub-scale scores. $\kappa_{\mathrm{w}}$ values of 0.4 or less were considered as poor; $0.4-0.6$ as fair to moderate; $0.6-0.8$ as good; and $>0.8$ as excellent inter-observer agreement. We planned to assess inter-rater reliability in $20 \%$ of the patients' sample and hence randomly reassessed one patient in every five patients' block. For these patients, the ICU nurse or ICU physician evaluator in the pair blindly scored FOUR, GLS scores within an hour. Third, outcome was assessed at 3 months using the Glasgow Outcome Scale (GOS) [24]. Poor outcome was defined as a GOS of 3 or less. A logistic regression analysis adjusted for age and etiology of coma was performed to assess the link between the studied scales and the outcome. The area under the receiver operating characteristic (ROC) curve was calculated to assess each model discrimination capability. Data were analyzed using Stata 11.1 (StataCorp. 2009. Stata Statistical Software: Release 11. College Station, TX: StataCorp LP). The study was approved by the University Medical Faculty Ethics 
Committee, and written informed consent was obtained by the patients' legal representative.

\section{Results}

176 acutely brain-damaged patients were included in our study (mean age $63 \pm 15$ years, range 18-87; 96 males; median time since ICU admission 8, range 46). Etiology was ischemic or hemorrhagic stroke ( $n=52$ patients), post anoxic-ischemic encephalopathy $(n=33)$, traumatic head injury $(n=22)$, central nervous system infection $(n=13)$, metabolic encephalopathy $(n=9)$, seizures and status epilepticus $(n=8)$, subarachnoid hemorrhage $(n=7)$, and miscellaneous acute neurological conditions $(n=32)$. Since the sample size was limited, we decided to categorize etiologies into traumatic and non-traumatic according to the Multi-Society Task Force on PVS [25]. Hundred and thirty-one patients were intubated at the time of assessment (74\%) and hence were scored 1 for the GLS/GCS verbal sub-score (15 non-intubated patients showed a "genuine" score of 1 on this GLS/GCS sub-scale). The frequency distribution of the 176 FOUR score and GLS/GCS ratings are displayed in Fig. 1. FOUR total score correlated with GCS and GLS total scores $(r=0.81, P<0.001$ and $r=0.82, P<0.001$, respectively).

The inter-rater agreement for the FOUR total score was $\operatorname{good}\left(\kappa_{\mathrm{w}}=0.75\right)$. For the agreement of each sub-scale, kappa values were good for visual $\left(\kappa_{\mathrm{w}}=0.80\right)$, fair to moderate for motor $\left(\kappa_{\mathrm{w}}=0.59\right)$, good brainstem $\left(\kappa_{\mathrm{w}}=\right.$ $0.77)$, and respiration sub-scales $\left(\kappa_{\mathrm{w}}=0.74\right)$. Agreement for the GCS and GLS total scores was good $\left(\kappa_{\mathrm{w}}=0.68\right.$ and 0.66 , respectively). Kappa values were good for the eyes $\left(\kappa_{\mathrm{w}}=0.68\right)$, motor $\left(\kappa_{\mathrm{w}}=0.69\right)$, and brainstem subscales $\left(\kappa_{\mathrm{w}}=0.73\right)$ but fair to moderate for the verbal $\left(\kappa_{\mathrm{w}}=0.56\right)$ sub-scale.

Outcome 3 months after acute brain insult was obtained in 136 patients (23\% missing data) (Table 1). Characteristics (age, etiology, GLS, GCS, and FOUR total scores) of patients with missing outcome were not different from those in whom outcome data were available (Table 2). Considering the FOUR score total score, 1-point increase in total score was associated with a $17 \%$ decrease of the odds ratio for poor outcome. This was also observed after adjusting for age and etiology (traumatic vs. non-traumatic). Similarly, for every 1-point increase in GCS total score, there was an odds ratio reduction of $19 \%$ of experiencing poor outcome under the unadjusted model. This relation remained after adjusting for age and etiology (traumatic vs. non-traumatic). For the GLS total score, there was an estimated odds ratio reduction of $19 \%$ of experiencing poor outcome under the unadjusted and the adjusted (age and etiology) model. Table 1 shows the relations between total scores and patients' outcome for each of the three scales.

Association between FOUR, GCS, GLS sub-scales, and outcome was assessed using stepwise backward logistic regressions. For the FOUR score, sub-scales associated with outcome were brainstem reflexes but not respiration. The only GCS sub-scales associated with outcome was the verbal sub-score even when adjusted for ventilation. The brainstem component of the GLS score was not associated with outcome; however, no patient with an absent pupillary reflex showed a good outcome in the present cohort. No patient with a FOUR score of 0 or 1 survived $(n=6)$, while 3 out of 15 patients with a GCS total score of 3 were alive after 3 months. However, no significant difference was found between the two scales $(P=0.53)$. ROC curves were estimated to compare prediction of poor outcome between the three scales. The area under the ROC curve (AUROC) were equivalent for the GCS versus the FOUR (AUROC $=0.68$ and 0.70, respectively, for GCS and FOUR, $P=0.67)$ and for the GLS versus the FOUR (AUROC $=0.72$ and 0.70 , respectively, for GLS and FOUR, $P=0.73$ ), but not for the GCS versus the GLS (AUROC $=0.68$ and 0.72 , respectively, for GCS and GLS, $P=0.006$ ).

In terms of clinical diagnosis, 71 included patients were considered as being in a vegetative/unresponsive state based on GCS assessment (i.e., GCS sub-scores showing spontaneous or stimulation-induced eye opening $\mathrm{E}>1$; absence of verbalization $\mathrm{V}<3$; and absence of localization of pain $M<5$ ). The FOUR score identified 8 of these 71 patients $(11 \%)$ as being minimally conscious given that they showed visual pursuit (FOUR sub-score Eye $=4$ ).

\section{Discussion}

In order to overcome deficiencies of the GLS/GCS, the FOUR score has been designed to provide further neurological details in coma patients, recognize certain unconscious states, and predict outcome (e.g., [23, 26].). Our study shows a good concurrent validity between the FOUR score and validated behavioral scales as the GCS and the GLS, in line with previous findings [23, 27-29]. Including the GLS in our coma scale assessment could be regarded of limited interest since this scale is not frequently used in clinical practice outside of French-speaking countries. However, before the FOUR was launched GLS was one of the rare, if not the only, coma scale including brainstem assessment. Our results pertain to a sample of severely brain-damaged patients (GCS $<8$ on admission) in contrast to other studies validating the FOUR in moderate or mild brain damage [23] [26, 29]. Even if it is a basic rule to assess patients free of any drug influence, the 

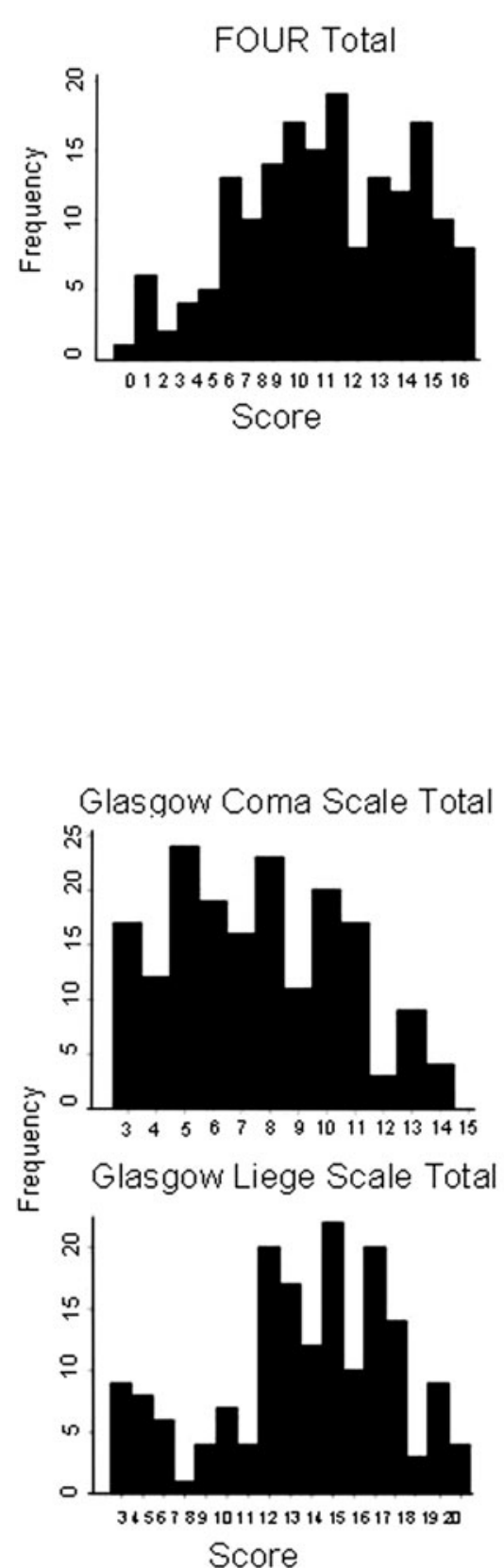

FOUR Score
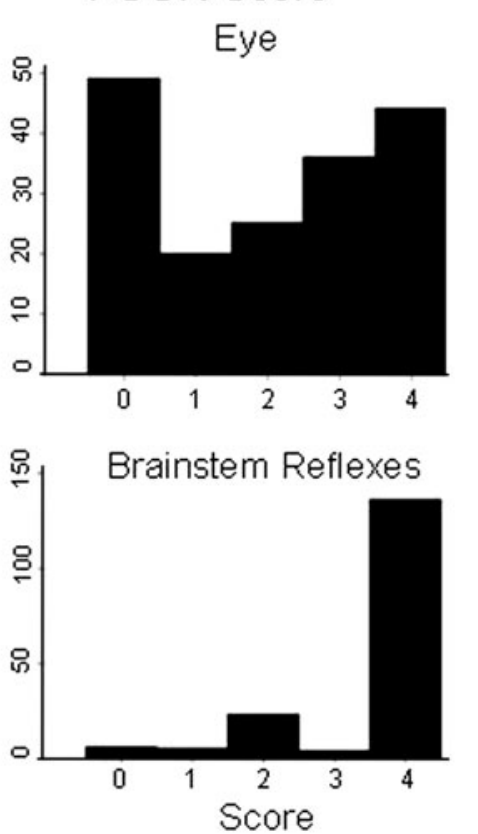

GCS/GLS
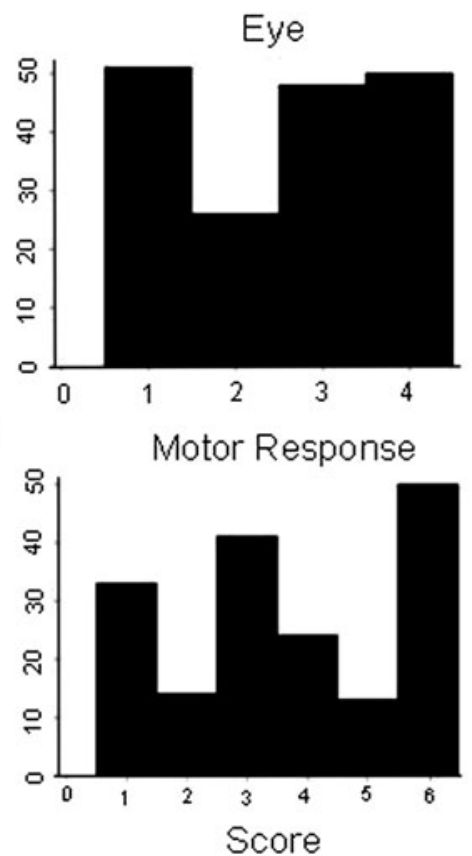

Respiration
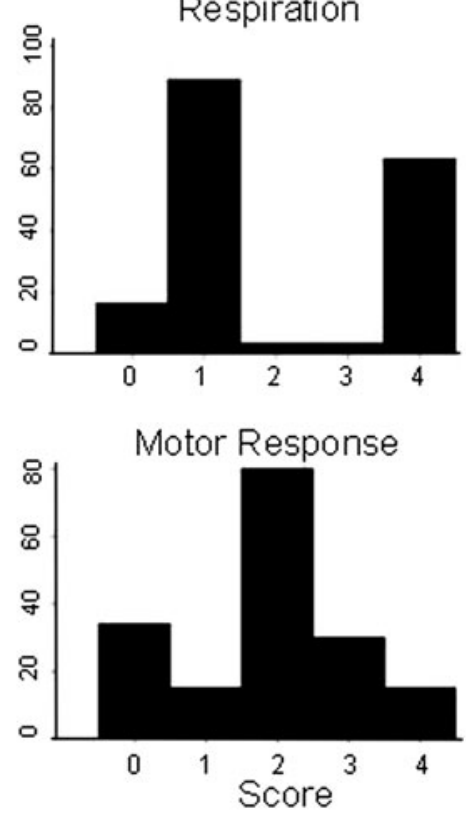

Verbal Response

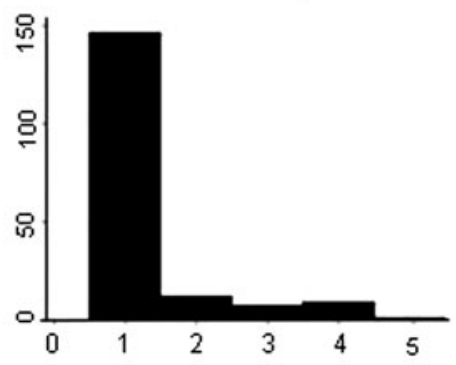

Brainstem Reflexes

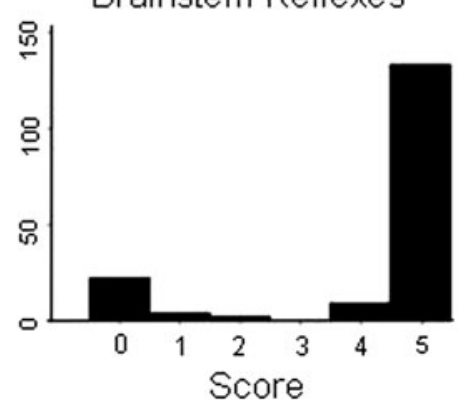

Fig. 1 Distribution of Full Outline of UnResponsiveness (FOUR), Glasgow Coma Scale (GCS), and Glasgow Liege Scale (GLS) sub-scores: eye, motor and verbal responses, brainstem reflexes, and respiration pattern

exclusion of patients receiving sedation or neuromuscular blocking agents could have introduced some bias and limit the generalizability of our conclusions. On the contrary including sedated patients would have led to overestimating patients' severity, bias their prognosis and hence negatively influence clinician attitudes. The reported results confirm good inter-rater reliability between ICU nurses and ICU physicians for the FOUR total score, corroborating previous studies showing a good to excellent inter-rater reliability between ICU physicians raters pairs [23, 30], trained and not trained ICU or neuroscience nurses [26, 29-31], non-neurology staff [32], medical intensivists, fellows and consultants pairs [29], and expert or novice clinicians and nurses pairs [27, 28]. It should 
Table 1 Comparison of outcome predictions (Glasgow Outcome Scale (GOS) poor outcome at 3 months) by the Full Outline of

UnResponsiveness score (FOUR), the Glasgow Coma Scale (GCS), and the Glasgow Liege Scale (GLS)

\begin{tabular}{|c|c|c|c|c|}
\hline & \multicolumn{2}{|c|}{ GOS poor outcome at 3 months } & \multicolumn{2}{|c|}{ Adjusted for age and etiology } \\
\hline & OR $(95 \% \mathrm{CI})$ & $P$ value & OR $(95 \% \mathrm{CI})$ & $P$ value \\
\hline FOUR score & $0.83(0.74-0.93)$ & 0.002 & $0.83(0.73-0.95)$ & 0.002 \\
\hline Eye & $0.75(0.57-0.97)$ & 0.029 & $0.75(0.58-0.99)$ & 0.041 \\
\hline Motor & $0.62(0.43-0.90)$ & 0.011 & $0.63(0.44-0.92)$ & 0.016 \\
\hline Brainstem & $0.51(0.27-0.98)$ & 0.043 & $0.46(0.22-0.95)$ & 0.037 \\
\hline Respiration & $0.71(0.54-0.92)$ & 0.011 & $0.71(0.54-0.93)$ & 0.013 \\
\hline GCS score & $0.81(0.71-0.93)$ & 0.003 & $0.82(0.71-0.95)$ & 0.007 \\
\hline GLS score & $0.81(0.72-0.91)$ & 0.001 & $0.81(0.71-0.92)$ & 0.001 \\
\hline Eye & $0.86(0.61-1.20)$ & 0.360 & $0.86(0.60-1.22)$ & 0.386 \\
\hline Motor & $0.75(0.6-0.94)$ & 0.014 & $0.77(0.61-0.98)$ & 0.032 \\
\hline Verbal & $0.44(0.29-0.69)$ & $<0.001$ & $0.45(0.29-0.71)$ & 0.001 \\
\hline Brainstem & $0.25(0.04-1.53)$ & 0.135 & $0.18(0.02-1.59)$ & 0.122 \\
\hline
\end{tabular}

Table 2 The patients' characteristics (age, etiology, interval, GCS total score, and FOUR total score) with missing outcome were not different from those where outcome data were available

\begin{tabular}{llll}
\hline & Non missing & Missing & $P$ value \\
\hline Age & $63.3 \pm 15.2$ & $63.9 \pm 14.2$ & 0.81 \\
Etiology (Traumatic) & $39 / 138(28 \%)$ & $10 / 29(26 \%)$ & 0.145 \\
FOUR & $9(6 ; 12)$ & $10(8 ; 14)$ & 0.10 \\
GLS & $12(9 ; 15)$ & $13(11 ; 15)$ & 0.08 \\
GCS & $7(5 ; 10)$ & $8(6 ; 10)$ & 0.10 \\
\hline
\end{tabular}

however be pointed that our study is limited by the small patient's sample in which inter-rater reliability was assessed. Of all the FOUR's sub-scales, the motor sub-scale inter-rater agreement was shown to be lowest (i.e., $\kappa_{\mathrm{w}}$ of 0.59 reflecting fair to moderate raters' agreement) whereas some studies on the GGS have shown that its motor component is the most reliable [33]. Similar observations were reported by previous studies using the FOUR score assessing agreement between novice clinicians $\left(\kappa_{\mathrm{w}}=0.54\right)$ and experienced nurses $\left(\kappa_{\mathrm{w}}=0.55\right)$ in neurosurgical patients [27]. It could be proposed that the presence of variable motor apraxia in some patients could influence the scoring of hand-coded command following. The possible bias of apraxia in the assessment of consciousness remains very challenging and requires further study. In addition, the different scoring of stereotyped motor responses to noxious stimulation in the FOUR as compared to the GLS/GCS (i.e., M2 is scored for stereotyped bilateral flexion posturing and unilateral (pathological or normal) flexion) might also explain the observed between-rater variability. As said, the FOUR score unlike the GLS/GLS does not require a verbal response, and thus may be more valuable in intensive care departments practices that typically have a large number of critically ill patients who have undergone intubation and cannot manifest a verbal response. Indeed, $74 \%$ of our study sample was intubated, implying that the verbal sub-scale of the GLS/GCS could not be scored. Similar to previous studies [23, 29] on FOUR respiration assessment, very few 2 and 3 scores (i.e., Cheyne Stokes and irregular breathing, respectively) were observed in the present cohort. It remains to be shown if this finding truly reflects the uncommonness of these respiratory patterns or if these could be related to suboptimal knowledge and scoring of patients' respiration.

In line with previous studies, FOUR and GLS/GCS total scores were comparable in predicting outcome $[23,26,29$ $32,34]$. In addition, no patient with a FOUR score of 0 or 1 survived, while $20 \%$ of patients with a GCS total score of 3 were alive at 3 month follow-up. Although this finding does not reach statistical significance, it corroborates previous studies $[30,35]$. The GLS was shown to herald superior outcome prediction as compared to the GCS [36, 37], but the GLS was here not shown to be superior to the prognostic capacity of the FOUR.

It is important to stress that the FOUR, unlike the GLS/ GCS, assesses eye tracking, one of the first signs heralding recovery of consciousness after coma and the vegetative/ unresponsive state [3]. The vegetative/unresponsive state is a clinical diagnosis that does not require temporal criteria $[38,39]$. The condition is called persistent when it persists for over 1 month (and permanent if over 3 months for nontraumatic and 12 months for traumatic etiology) [40]. Patients who fail to show signs of consciousness (i.e., command following or non-reflex movements) but do show eye opening (spontaneous or induced) are no longer in coma but are in vegetative/unresponsive state. Once voluntary movements or command following is observed but no functional communication can be established patients are now defined as being in a minimally conscious state [3]. Based on GLS/GCS and FOUR assessments, vegetative/unresponsive state was defined by GLS/GCS score of $\mathrm{E}>1, \mathrm{~V}<3, \mathrm{M}<5$, and FOUR score showing $\mathrm{E}<4$, $\mathrm{M}<3$. The diagnosis of minimally conscious [41] and 
locked-in states [42] may be very challenging, especially in the acute setting. In the studied sample $40 \%(N=71)$ of patients were "vegetative" (i.e., showed wakeful unawareness) based on GLS/GCS assessment. However, by identifying the presence of visual pursuit, the FOUR score showed that this diagnosis was erroneous in $11 \%$ (8 out of 71 patients) of these patients. Disentangling vegetative/ unresponsive from minimally conscious state is of key medical and ethical importance [43] as functional neuroimaging [6] and behavioral [44] data have shown evidence for residual pain perception and emotional processing in the latter condition. The possible consequence of considering patients as unconscious (while they actually show (minimal) signs of consciousness) could have clinical consequences, for example, in terms of pain and symptom management. In our sample, the use of the FOUR could have permitted to treat for pain in about 1 in 10 of ICU patients $(8 / 71)$ otherwise possibly considered as insensate and vegetative/unresponsive. Hence, the routine clinical use of the FOUR score may permit to identify as soon as possible minimal (non-verbal) signs of consciousness, permitting to assure early and appropriate pain and symptom management in these challenging non-communicative ICU patients $[45,46]$.

In conclusion, our prospective study comparing the FOUR, GLS/GCS scores in patients who are severely brain damaged shows that the FOUR is a valid tool with prognostic value comparable to GCS and GLS as here demonstrated by the AUC data from ROC analyses. The FOUR score may offer the additional advantage to be performable in intubated patients and to identify nonverbal signs of consciousness by assessing visual pursuit.

\section{References}

1. Jennett B, Plum F. Persistent vegetative state after brain damage. A syndrome in search of a name. Lancet. 1972;1:734-7.

2. Laureys S, Celesia GG, Cohadon F, et al. Unresponsive wakefulness syndrome: a new name for the vegetative state or apallic syndrome. BMC Med. 2010;8:68.

3. Giacino JT, Ashwal S, Childs N, et al. The minimally conscious state: definition and diagnostic criteria. Neurology. 2002;58:349-53.

4. Schnakers C, Chatelle C, Majerus S, Gosseries O, De Val M, Laureys S. Assessment and detection of pain in noncommunicative severely brain-injured patients. Expert Rev Neurother. 2010;10:1725-31.

5. Demertzi A, Schnakers C, Ledoux D, et al. Different beliefs about pain perception in the vegetative and minimally conscious states: a European survey of medical and paramedical professionals. Prog Brain Res. 2009;177:329-38.

6. Boly M, Faymonville ME, Schnakers C, et al. Perception of pain in the minimally conscious state with PET activation: an observational study. Lancet Neurol. 2008;7:1013-20.

7. Schiff ND, Rodriguez-Moreno D, Kamal A, et al. fMRI reveals large-scale network activation in minimally conscious patients. Neurology. 2005;64:514-23.
8. Laureys S, Perrin F, Bredart S. Self-consciousness in non-communicative patients. Conscious Cogn. 2007;16:722-41. discussion 742-725.

9. Teasdale G, Jennett B. Assessment of coma and impaired consciousness. A practical scale. Lancet. 1974;2:81-4.

10. Laureys S, Piret S, Ledoux D. Quantifying consciousness. Lancet Neurol. 2005;4:789-90.

11. Rordorf G, Koroshetz W, Efird JT, Cramer SC. Predictors of mortality in stroke patients admitted to an intensive care unit. Crit Care Med. 2000;28:1301-5.

12. Gotoh O, Tamura A, Yasui N, Suzuki A, Hadeishi H, Sano K. Glasgow Coma Scale in the prediction of outcome after early aneurysm surgery. Neurosurgery. 1996;39:19-24. discussion $24-15$.

13. Tien HC, Cunha JR, Wu SN, et al. Do trauma patients with a Glasgow Coma Scale score of 3 and bilateral fixed and dilated pupils have any chance of survival? J Trauma. 2006;60:274-8.

14. Stocchetti N, Citerio G, Maas A, Andrews P, Teasdale G. Bryan Jennett and the field of traumatic brain injury. His intellectual and ethical heritage in neuro-intensive care. Intensive Care Med. 2008;34:1774-8.

15. Rowley G, Fielding K. Reliability and accuracy of the Glasgow Coma Scale with experienced and inexperienced users. Lancet. 1991;337:535-8.

16. Murray GD, Teasdale GM, Braakman R, et al. The European Brain Injury Consortium survey of head injuries. Acta Neurochir (Wien). 1999;141:223-36.

17. Marion DW, Carlier PM. Problems with initial Glasgow Coma Scale assessment caused by prehospital treatment of patients with head injuries: results of a national survey. J Trauma. 1994; 36:89-95.

18. Born JD, Hans P, Dexters G, et al. Practical assessment of brain dysfunction in severe head trauma. Neurochirurgie. 1982;28:1-7.

19. Balestreri M, Czosnyka M, Chatfield DA, et al. Predictive value of Glasgow Coma Scale after brain trauma: change in trend over the past ten years. J Neurol Neurosurg Psychiatry. 2004;75:161-2.

20. Benzer A, Mitterschiffthaler G, Marosi M, et al. Prediction of non-survival after trauma: Innsbruck Coma Scale. Lancet. 1991;338:977-8.

21. Starmark JE, Stalhammar D, Holmgren E, Rosander B. A comparison of the Glasgow Coma Scale and the Reaction Level Scale (RLS85). J Neurosurg. 1988;69:699-706.

22. Gill M, Windemuth R, Steele R, Green SM. A comparison of the Glasgow Coma Scale score to simplified alternative scores for the prediction of traumatic brain injury outcomes. Ann Emerg Med. 2005;45:37-42.

23. Wijdicks EF, Bamlet WR, Maramattom BV, Manno EM, McClelland RL. Validation of a new coma scale: the FOUR score. Ann Neurol. 2005;58:585-93.

24. Jennett B, Bond M. Assessment of outcome after severe brain damage. Lancet. 1975;1:480-4.

25. The Multi-Society Task Force on PVS. Medical aspects of the persistent vegetative state (2). N Engl J Med 1994;330: 1572-1579.

26. Wolf CA, Wijdicks EF, Bamlet WR, McClelland RL. Further validation of the FOUR score coma scale by intensive care nurses. Mayo Clin Proc. 2007;82:435-8.

27. Akavipat P. Endorsement of the FOUR score for consciousness assessment in neurosurgical patients. Neurol Med Chir (Tokyo). 2009;49:565-71.

28. Idrovo L, Fuentes B, Medina J, et al. Validation of the FOUR Score (Spanish Version) in acute stroke: an interobserver variability study. Eur Neurol. 2010;63:364-9.

29. Iyer VN, Mandrekar JN, Danielson RD, Zubkov AY, Elmer JL, Wijdicks EF. Validity of the FOUR score coma scale in the medical intensive care unit. Mayo Clin Proc. 2009;84:694-701. 
30. Fischer M, Ruegg S, Czaplinski A, et al. Inter-rater reliability of the Full Outline of UnResponsiveness score and the Glasgow Coma Scale in critically ill patients: a prospective observational study. Crit Care. 2010;14:R64.

31. Cohen J. Interrater reliability and predictive validity of the FOUR score coma scale in a pediatric population. J Neurosci Nurs. 2009;41:261-7. quiz 268-9.

32. Stead LG, Wijdicks EF, Bhagra A, et al. Validation of a new coma scale, the FOUR score, in the emergency department. Neurocrit Care. 2009;10:50-4.

33. Healey C, Osler TM, Rogers FB, et al. Improving the Glasgow Coma Scale score: motor score alone is a better predictor. J Trauma. 2003;54:671-8. discussion 678-80.

34. Eken C, Kartal M, Bacanli A, Eray O. Comparison of the Full Outline of Unresponsiveness Score Coma Scale and the Glasgow Coma Scale in an emergency setting population. Eur J Emerg Med. 2009;16:29-36.

35. Fugate JE, Rabinstein AA, Claassen DO, White RD, Wijdicks EF. The FOUR score predicts outcome in patients after cardiac arrest. Neurocrit Care. 2010;13:205-10.

36. Born JD. The Glasgow-Liège Scale. Prognostic value and evaluation of motor response and brain stem reflexes after severe head injury. Acta Neurochir. 1988;95:49-52.

37. Born JD, Albert A, Hans P, Bonnal J. Relative prognostic value of best motor response and brain stem reflexes in patients with severe head injury. Neurosurgery. 1985;16:595-601.
38. Jennett B. Thirty years of the vegetative state: clinical, ethical and legal problems. Prog Brain Res. 2005;150:537-43.

39. Laureys S, Boly M. What is it like to be vegetative or minimally conscious? Curr Opin Neurol. 2007;20:609-13.

40. The Multi-Society Task Force on PVS. Medical aspects of the persistent vegetative state (1). N Engl J Med 1994;330: 1499-1508.

41. Schnakers C, Vanhaudenhuyse A, Giacino J, et al. Diagnostic accuracy of the vegetative and minimally conscious state: clinical consensus versus standardized neurobehavioral assessment. BMC Neurol. 2009;9:35.

42. Laureys S, Pellas F, Van Eeckhout P, et al. The locked-in syndrome: what is it like to be conscious but paralyzed and voiceless? Prog Brain Res. 2005;150:495-511.

43. Bernat JL. Current controversies in states of chronic unconsciousness. Neurology. 2010;75:S33-8.

44. Schnakers C, Chatelle C, Vanhaudenhuyse A, et al. The Nociception Coma Scale: a new tool to assess nociception in disorders of consciousness. Pain. 2010;148:215-9.

45. Schnakers C, Zasler ND. Pain assessment and management in disorders of consciousness. Curr Opin Neurol. 2007;20:620-6.

46. Bruno MA, Gosseries O, Ledoux D, Hustinx R, Laureys S. Assessment of consciousness with electrophysiological and neurological imaging techniques. Curr Opin Crit Care. 2011;17(2): 146-57. 